\title{
A propos de l'assistance aux mourants
}

\author{
Philippe Gauer
}

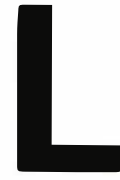

e 29 avril 1991, une proposition de résolution "sur l'assistance aux mourants" était présentée devant le Parlement européen par le professeur Léon Schwarzenberg au nom de la commission de l'Environnement, de la Santé publique et de la Protection des consommateurs.

Ce rapport*, qui a été approuvé par 16 voix contre 11 et 3 abstentions, est actuellement soumis à l'examen de la commission juridique du Parlement européen, en attendant que le texte définitif soit présenté en séance plénière en 1992.

Il est très intéressant de voir comment un texte faisant l'apologie de l'euthanasie sous sa forme active a pu être adopté. Après le droit de tuer un enfant dans le sein de sa mère en cas de détresse, allons-nous avoir le droit de tuer le malade en cas de souffrances à l'approche de la fin de sa vie ? Cette proposition de résolution se compose de 12 considérants et de 8 conclusions dont allons retenir quelques exemples significatifs.

Parmi les premiers considérants, nous découvrons quelques définitions comme la suivante : "le fondement de la vie d'un être humain est la dignité** et la spiritualité, et donc l'existence d'une personne n'est pas réductible aux fonctions naturelles, c'est-à-dire aux fonc-

\footnotetext{
* Proposition de résolution "sur l'assistance aux mourants ". Rapport de la commission de l'Environnement, de la Santé publique et de la Protection des consommateurs (29 avnil 1991). Documentation Catholique $1991 ; 2034$ : 791-3

$\mathrm{m} / \mathrm{s} n^{\circ} 1$, vol. 8 , janvier 92
}

tions de la vie végétative. Ces affirmations nous sont proposées dès le début du texte dans un contexte philosophique assez confus. Ainsi, si le concept de "dignité " peut être rattaché à la personne humaine, c'est en tant que droit de sa personne - comme élément constitutif des Droits de l'homme - dans ses rapports avec les autres hommes et non pas en tant que fondement de son être. Chaque homme doit être traité dignement de par sa nature humaine, c'est-à-dire respecté dans ce qu'il est et dans ce qu'il pense. La dignité humaine ne fonde pas un être humain, mais est un droit essentiel de cet être vivant qui est un homme. Rattacher le concept de spiritualité au fondement d'un être humain dans un tel texte entraîne la même confusion dans les esprits. Si beaucoup de courants philosophiques reconnaissent à l'homme une dimension spirituelle, ce n'est ni une constante ni un dogme. De plus, ce concept prend des significations tellement variables et parfois contradictoires (il suffit de comparer la pensée judéochrétienne avec celle du new age, par exemple, pour s'en convaincre) qu'il demande à être explicité et précisé. Après cette double confusion - dignité et spiritualité - sur le fondement de la vie d'un être humain, le texte égare le lecteur encore davantage...

Le troisième considérant est fait de deux propositions : "Le fonctionnement cérébral détermine le niveau de conscience" et "le niveau de conscience définit un être humain ". Ces deux propositions prises séparément sont toutes les deux acceptables, mais moyennant une précision capitale absente du texte: celle du contexte sémantique dans lequel elles sont insérées. Ainsi, affirmer que "le fonctionnement cérébral détermine le niveau de conscience " est une proposition d'ordre biologique ou médical, et qui concerne le fonctionnement neurologique, alors qu'affirmer que " le niveau de conscience définit un être humain " relève d'un discours anthropologique et cherche plutôt à situer la place de l'homme dans le règne animal. Nous devons donc nous interroger sur le sens de la juxtaposition de ces deux propositions vraies mais de nature différente : leur utilisation respectivement comme la majeure et la mineure d'un syllogisme ne peut conduire qu'à un sophisme comme nous le verrons dans la huitième conclusion !

Le douzième considérant (L) mérite l'attention. Nous y lisons que "l'aspiration à un sommeil définitif ne constitue pas une négation de la vie mais la demande d'interruption d'une existence à laquelle la maladie a fini par ôter toute dignité ". Nous découvrons là un merveilleux jeu de paraphrases autour du sujet de la " mort ", qui est successivement qualifiée de "sommeil définitif " puis d' "interruption d'une existence " dont il est d'ailleurs nié qu'il s'agit d'une "négation de la vie ». C'est dans ce contexte qu'il faut essayer de comprendre le sens précis de la fin de la phrase : " une existence à laquelle la maladie a fini par ôter toute dignité ". Cette affirmation doit être relue naturellement - si nous considérons qu'il y a une logique interne à ce rapport à la lumière du 
premicr considérant, où on lisait quc " lc fondement de la vie d'un êtrc humain est la dignitć et la spiritualité " et quc "la vie se définit par la dignité ". Une existence qui a perdu sa dignité est donc unc vic qui a perdu son fondement et sa définition même. Nous ne sommes donc plus en face d'un vivant mais d'un mort, tout au plus d'un être vivant ayant perdu toutc conscience et donc dans un état de coma dépassé. Comment pcut-on parler alors d'aspiration de cettc même personne à un sommcil définitif auquel la maladic l'a déjà conduite ?

Il est néccssairc de bien comprendre les considérants pour saisir les enjeux des conclusions qui suivent. La huitième, par cxemple, estime qu'" en l'absence de toutc thérapcutique curative, et après échec de soins palliatifs, correctement conduits tant sur lc plan psychologique que médical, et que chaque fois qu'un malade plcinement conscient demande, de manic̀re pressante ct continue, qu'il soit mis un terme à unc cxistence qui a pcrdu pour lui toutc dignité, et qu'un collège dc médecins constitué à dessein constatc l'impossibilité d'apporter de nouveaux soins spécifiques, cettc demande doit être satisfaisante sans qu'il soit, de cettc façon, porté atteintc au respect de la vic humaine ". Essayons de dégager les liens logiques de ce paragraphe que l'on peut ramencr à trois propositions : (1) un malade pleinement conscient demandc qu'il soit mis un terme à son existence ;

(2) unc existence qui a perdu pour lui toute dignité ;

(3) cette demande doit être satisfaite sans qu'il soit, de cette façon, porté attcinte au respect de la vie humaine. Rappclons que, dans le contexte de ce rapport, "la vie se définit par la dignité " ct quc "le niveau de conscience définit un être humain ". Il nous faut donc comprendre qu'une cxistence ayant perdu toutc dignité est cellc d'un être humain qui a perdu tout niveau de conscience et qui donc, comme l'affirme par aillcurs ce rapport, n'a plus d'activité cérébrale puisque "le fonctionnement cérébral déterminc lc niveau de conscience ". Cette huitième conclusion semblerait donc présenter une contradiction entre le membrc (1) "un malade conscient" et lc membre (2) "unc existence qui a perdu pour lui toute dignité ", si l'on oubliait la dimension subjective du rapport qu'il y a entre (1) et (2), car c'est "pour lui ", le malade, que l'existence scmble avoir perdu sa dignité. C'est sur cet aspect subjectif que nous devons concentrer notre attention, car là se trouvc lc nœud du faux syllogismc proposé par le texte. Si le maladc est conscient, c'est donc quc sa (propre) vie n'a pas perdu sa dignité, sclon la logique du texte. Il n'est donc pas question de pratiquer un acte d'euthanasic, ce serait un crimc. En revanche, si c'est un observatcur extérieur, médecin, clinicien... qui juge que la vie, pour ce maladelà, scmble avoir perdu toute dignité, deux cas peuvent se présenter. Le premier est celui d'un malade en coma dépassé, où, bien évidemment, interromprc une telle existence n'est effectivement pas porter atteinte au respect de la vie humainc, mais revient simplement à éviter un acharnement thérapeutique dans une situation vide de tout espoir médical. Le deuxième cas est celui d'un malade dont l'état peut être grabataire ou la conscience, plus ou moins altérée ; qu'est-ce qui permet ici à quelqu'un (même s'il est médecin !), d'affirmer que la personne qu'il a sous les yeux a perdu toute dignité et ne méritc plus de vivrc ? Qui peut se permettre d'avoir un droit de vic ou de mort sur quclqu'un, car, ne l'oublions pas, l'hypothèse de départ étant bien cellc d'un " malade plcincment conscient " et non celle d'une personne en coma dépassé ?

Cet énorme sophisme va permettre de justifier le droit de tuer les malades qui nous dérangent. Allons-nous accepter que la loi affurme qu'“ interrompre une existence... est le respect de la vie ".

\section{Philippe Gauer}

Docteur en médecine, 2, allée des Hirondelles, 54300 Luréville.

\section{TIRÉS A PART}

P. Gauer 